\title{
Quantification of small fiber pathology in patients with sarcoidosis and chronic pain using cornea confocal microscopy and skin biopsies
}

This article was published in the following Dove Press journal: Journal of Pain Research

26 August 2017

Number of times this article has been viewed

\author{
Linda CJ Oudejans' \\ Marieke Niesters' \\ Michael Brines ${ }^{2}$ \\ Albert Dahan' \\ Monique van Velzen' \\ 'Department of Anesthesiology, \\ Leiden University Medical Center, \\ Leiden, the Netherlands; ${ }^{2}$ Araim \\ Pharmaceuticals, Inc., Tarrytown, NY, \\ USA
}

\begin{abstract}
Small fiber pathology with concomitant chronic neuropathic pain is a common complication of sarcoidosis. The gold standard of diagnosis of small fiber neuropathy (SFN) is the quantification of small nerve fibers in skin biopsies in combination with patient history and psychophysical tests; a new technique is the quantification of small nerve fibers in the cornea using cornea confocal microscopy (CCM). Here, we studied small fiber morphology in sarcoidosis patients with neuropathic pain using skin biopsies, CCM, and quantitative sensory testing (QST). Our aim was to construct specific phenotypes of neuropathic pain in sarcoidosis. Fiftyeight patients with a confirmed diagnosis of sarcoidosis and with moderate-to-severe neuropathic pain were tested. Decreased intraepidermal nerve fiber density (IENFD) from skin biopsies was found in $28 \%$ of patients, and CCM abnormalities were observed in $45 \%$ of patients. There was no correlation between CCM and IENFD abnormalities. Eighty-three percent of patients had abnormal thermal detection thresholds, a sign of small fiber dysfunction. Based on the presence or absence of abnormalities in IENFD and CCM, four distinct phenotypes were identified with a distinct homogeneous pattern of somatosensory symptoms. We argue that these distinct phenotypes have a similar mechanistic construct with specific phenotype-specific treatment options. Additionally, our data suggest the presence of patients with length- and nonlength-dependent SFN within this population of sarcoidosis patients.
\end{abstract}

Keywords: chronic pain, sarcoidosis, small fiber neuropathy

\section{Plain language summary}

Patients with chronic pain due to damaged small nerve fibers (neuropathic pain) are difficult to diagnose and treat adequately. The gold standard is to count the number of small nerve fibers in a piece of skin (biopsy), but the relatively novel technique of cornea confocal microscopy (CCM) can also be used to count the number of small nerve fibers (in the eye). In this study, we tested 58 sarcoidosis patients (an inflammatory disease) with neuropathic pain using skin biopsies and CCM, as well as other tests of nerve fiber function. We found that $28 \%$ of patients had an abnormal skin biopsy, indicating small nerve fiber damage, and $45 \%$ of patients showed abnormal nerve fibers using the CCM technique. The majority of patients (83\%) displayed abnormal nerve fiber function tests. We identified four subgroups of patients, based on the presence or absence of skin biopsy and CCM abnormalities. These findings suggest that the sarcoidosis patient population with neuropathic pain is heterogeneous, and subgroups can be constructed that likely require different treatment strategies.

\section{Introduction}

Small fiber neuropathy (SFN) is a complication of various disorders with metabolic and/or inflammatory components such as diabetes mellitus and sarcoidosis. SFN is
Correspondence: Albert Dahan Department of Anesthesiology, Leiden University Medical Center, H5-P, 2300 RC, Leiden, the Netherlands

$\mathrm{Tel}+3$ I 7I 526230 I

Fax +31 71 5266230

Email a.dahan@lumc.nl 
often associated with severe chronic, neuropathic pain, which significantly impacts the quality of life. Treatment is generally directed toward the pathogenesis of the underlying disease and the management of pain. Diagnostic and psychophysical tests for neuropathy such as skin biopsies, nerve conduction studies, and quantitative sensory testing (QST) can be used to construct patient-specific neuropathy phenotypes. As we published earlier, ${ }^{1}$ phenotyping may be applied for two specific reasons: first, phenotyping serves as a tool to better understand the disease process mechanistically and in terms of severity and, second, it may be used in the choice of treatment and consequently shorten the time until adequate pain relief. Currently, treatment is based on a trial-and-error approach with limited efficacy in a limited number of patients. ${ }^{2-5}$

SFN is prevalent in a large proportion of patients with sarcoidosis. Sarcoidosis is a complex inflammatory disease with pulmonary symptoms in a large proportion of patients. Additionally, chronic pain with signs of SFN is present in up to $70 \%$ of sarcoidosis patients. ${ }^{6-9}$ Previously, diagnosis of SFN has relied on the assessment of nerve fiber densities in skin biopsies; however, the relatively new technique of cornea confocal microscopy (CCM) is being used increasingly to quantify nerve fibers in the cornea to detect small fiber pathology. ${ }^{10,11}$ In the current study, we quantified the small fibers of the skin and cornea in sarcoidosis patients with chronic pain to determine whether specific phenotypes related to small fiber pathology exist. Additionally, we performed $\mathrm{QST}^{12}$ to further refine the specific phenotypes that we detected from CCM and skin biopsies. We hypothesize that we would find a large proportion of sarcoidosis patients with both signs of small fiber pathology in skin biopsies and cornea nerve morphology. The observation of small fiber abnormalities on both locations has previously been observed in diabetes patients. ${ }^{13}$

\section{Methods}

\section{Ethics}

The study protocols were approved by the local Ethics Committee (Leiden University Medical Center, Leiden, the Netherlands). All study procedures were conducted according to GCP guidelines and adhered to the tenets of the Declaration of Helsinki. The patients gave verbal and written informed consent before the start of the study. Data were selected from two Phase II trials, which are registered in the Netherlands Trial Register, identifiers NTR3575 and NTR4260.

\section{Patient selection}

Patients with a confirmed diagnosis of sarcoidosis were approached for participation in the study. All patients suffered from chronic sarcoidosis and were clinically in remission at the time of the study. The following inclusion criteria applied: 1) age between 18 and 70 years; 2) body mass index (BMI) $<35 \mathrm{~kg} / \mathrm{m}^{2} ; 3$ ) a spontaneous pain level ("pain now" or "average daily pain") of $\geq 5$ on an 11-point numerical rating scale $(0=$ no pain and $10=$ worst pain imaginable $) ; 4)$ pain defined as distal pain/discomfort with one of the following: dysesthesia, burning/painful feet worsening at night, and intolerance of sheets or clothes touching the legs or feet; and 5) a score of $>22$ on the SFN screening list (SFNSL) questionnaire. A spontaneous pain level of $<5$ was acceptable with an SFNSL score of $>37$. The SFNSL is a validated questionnaire used to detect the presence of SFN in the Dutch population. ${ }^{14}$ Patients were allowed to continue their medication for sarcoidosis and/or neuropathic pain; the most common types are listed in Table 1.

\section{Skin biopsies}

Skin biopsies were obtained from the distal leg $(10 \mathrm{~cm}$ above the lateral malleolus) by using a disposable punch biopsy (3 mm) and processed following established guidelines. ${ }^{15} \mathrm{~A}$ detailed description of the methods can be found in Dahan et al. ${ }^{16}$ Sex- and age-dependent normative data of nerve fiber density used for the distal leg were those of Lauria et al. ${ }^{17}$ Here, we report the intraepidermal nerve fiber density (IENFD). All measurements and counting were performed by a single, blinded individual.

\section{CCM}

Cornea nerve fiber density (CNFD), cornea nerve fiber length (CNFL), and cornea nerve branching density (CNBD) were determined using the Rostock Cornea Module with the Heidelberg Retina Tomograph III (Heidelberg, Germany) (for a full description of CCM methodology, refer Dahan et al. ${ }^{16}$ In short, after topical anesthesia of the eyes, the microscope was

Table I Patients' characteristics

\begin{tabular}{ll}
\hline Number of patients, $\mathrm{n}$ (male/female) & $58(3 \mathrm{I} / 27)$ \\
Age in years, mean (range) & $50(26-68)$ \\
Body mass index, $\mathrm{kg} / \mathrm{m}^{2}$, mean (range) & $25.8(18.4-32.7)$ \\
Years since diagnosis, mean (range) & $8.7(\mathrm{I}-37)$ \\
Patients on medication, $\mathrm{n}(\%)$ & $54(93)$ \\
Prednisone & $12(2 \mathrm{I})$ \\
Methotrexate & $12(2 \mathrm{I})$ \\
Folic acid & $9(16)$ \\
Acetaminophen & $19(33)$ \\
NSAIDs & $13(22)$ \\
Tramadol & $9(16)$ \\
Pregabalin & $11(19)$ \\
\hline
\end{tabular}

Abbreviation: NSAIDs, nonsteroidal anti-inflammatory drugs. 
placed at the surface of the cornea apex. Confocal images were acquired with a field of view of $400 \mu \mathrm{m} \times 400 \mu \mathrm{m}$ and automatically quantified using the ACCMetrics software. ${ }^{18-20}$ Three to eight representative, high-quality images were selected from a total of at least 50 images per eye, according to the recommendations. ${ }^{13,21} \mathrm{CNFD}, \mathrm{CNFL}$, and CNBD were quantified by an investigator who was blinded for subject demographics and disease state. Because a good correlation has been demonstrated between semiautomated (CCMetrics) and automated (ACCMetrics) corneal nerve fiber quantifications, ${ }^{13,22}$ data from the current study were compared with semiautomatically quantified reference values of age- and gender-matched healthy volunteers from Tavakoli et al. ${ }^{23}$

\section{QST}

Thermal detection and pain thresholds, mechanical detection and pain thresholds, pressure pain and vibration detection were evaluated on the following three district locations: the face, hand, and foot. All measurements were performed according to the protocol of the German research network on neuropathic pain, and data were expressed accordingly as transformed $z$-scores where values $<-1.96$ (loss of function) or $>+1.96$ (gain of function) are considered abnormal. ${ }^{12}$ Dynamic mechanical allodynia and paradoxical heat sensations were scored dichotomously as present or not present. Pain scoring was adapted in the protocol from a $0-100$ point scale to a $0-10$ point scale to simplify scoring and because the Dutch population is used to 11-point scoring systems in general (eg, in the clinic). Normative data were obtained from Magerl et al. ${ }^{24}$ Patients were considered to have abnormal sensations when at least one of the three anatomic locations deviated from normal values.

\section{Statistical analysis}

Statistical analyses were performed using the SPSS statistics Version 23 (IBM Corporation, Armonk, NY, USA) and GraphPad Prism 6 (GraphPad Software, Inc., La Jolla, CA, USA). Comparisons between groups were evaluated with the Kruskal-Wallis test (differences among phenotypes). Correlations of skin biopsy and CCM parameters were analyzed using linear regression. $P$-values $<0.05$ were considered significant. Data are presented as average $\pm 95 \%$ confidence interval (CI), unless otherwise indicated.

\section{Results \\ Patients}

A total of 58 patients with sarcoidosis (27 females, 47\%) were included in this study. Patients' characteristics are presented in Table 1 . The mean age was 50 years (range 26-68 years), the mean BMI was $26 \mathrm{~kg} / \mathrm{m}^{2}$ (range $18-33 \mathrm{~kg}$ / $\mathrm{m}^{2}$ ), and the mean sarcoidosis disease duration was 9 years (range 1-37 years).

\section{Skin biopsies}

Due to difficulties with processing of a skin tissue sample, the IENFD of one patient could not be determined. Average IENFD was $5.5 \mathrm{n} / \mathrm{mm}^{2}\left(95 \%\right.$ CI: $\left.5.16-5.84 \mathrm{n} / \mathrm{mm}^{2}\right)$. The percentage of patients in which IENFD values were $<5$ th percentile of the normative data of Lauria et a ${ }^{17}$ was 28. IENFD data are summarized in Table 2 and shown in Figure 1A. IENFD data are shown relative to the lower 5th percentile (averaged for men and women) of the 50-59-year age category $\mathrm{CI}$.

\section{CCM}

Since CCM data of the left and right eye were similar (data not shown), these data were averaged per patient. Mean CNFD, CNFL, and CNBD values are given in Table 2. Examples of patients with abnormal and normal cornea morphologies and a healthy male control are shown in Figure 2 for clarification. CNFD, CNFL, and CNBD values were $<5$ th percentile of reference values in 9\% (CNFD), 43\% (CNFL), and 28\% (CNBD). Compared to normative reference values, at least one abnormal parameter in cornea nerve morphology was observed in $45 \%$ of sarcoidosis patients. Individual scores of CCM parameters are illustrated in Figure 1B-D. Similar to the IENFD scores, the dotted line represents the lower 5 th percentile of the 56-65 years age category CI (averaged for men and women) of healthy controls. ${ }^{23}$

Table 2 Quantification of corneal nerve fibers by cornea confocal microscopy or skin biopsy in sarcoidosis patients

\begin{tabular}{llll}
\hline Nerve fiber parameter & Average $(\mathbf{9 5} \% \mathbf{C l})$ & Range & Significantly decreased, $\boldsymbol{n}(\%)$ \\
\hline IENFD $(\mathrm{n} / \mathrm{mm})$ & $5.5(5.16-5.84)$ & $0.8-11.7$ & $16(28)$ \\
CNFD $\left(\mathrm{n} / \mathrm{mm}^{2}\right)$ & $22.1(21.3-22.9)$ & $9.4-34.4$ & $5(9)$ \\
CNFL $\left(\mathrm{mm} / \mathrm{mm}^{2}\right)$ & $13.6(13.1-14.1)$ & $6.8-21.3$ & $25(43)$ \\
CNBD $\left(\mathrm{n} / \mathrm{mm}^{2}\right)$ & $32.5(29.8-35.2)$ & $3.1-84.4$ & $16(28)$ \\
\hline
\end{tabular}

Abbreviations: CNFD, cornea nerve fiber density; CNBD, cornea nerve branching density; CNFL, cornea nerve fiber length; IENFD: intraepidermal nerve fiber density. 
A

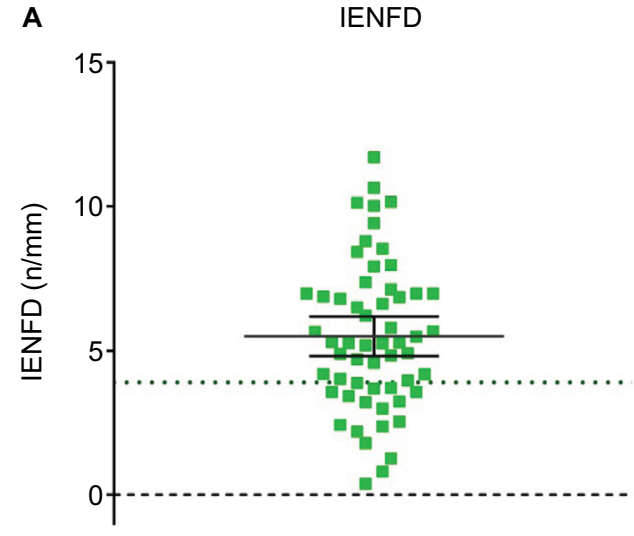

C

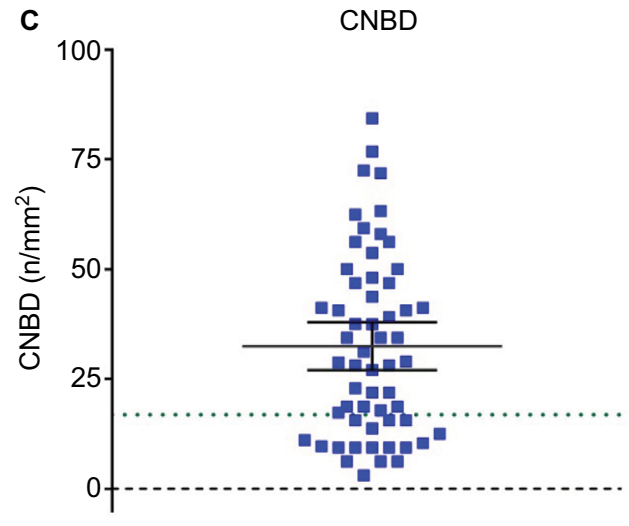

B

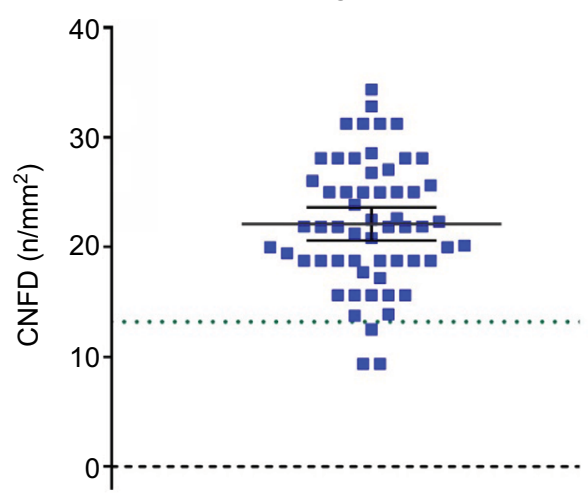

D

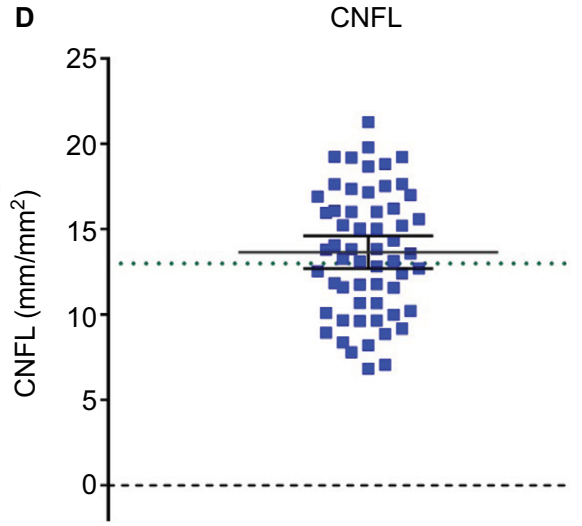

Figure I Average ( $95 \%$ confidence interval) intraepidermal nerve fiber density (IENFD) (A), cornea nerve fiber density (CNFD) (B), cornea nerve branching density (CNBD) (C), and cornea nerve fiber length (CNFL) (D) of sarcoidosis patients.

Note: The dotted line represents the 5 th percentile below which values are considered abnormal for the age category (46-65) of the average age of the patients.
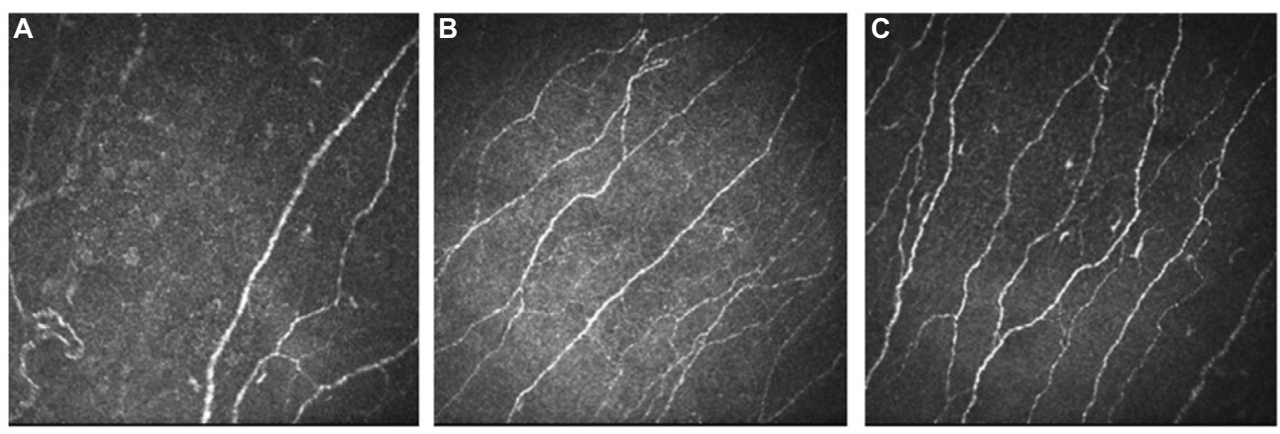

Figure 2 Confocal microscopy images of corneal nerve fibers of sarcoidosis patients with neuropathic pain and a healthy control.

Notes: (A) 52-year-old male sarcoidosis patient with abnormal corneal nerve fibers. (B) 53-Year-old male sarcoidosis patient with normal corneal nerve fiber state. (C) 53-year-old healthy male volunteer with normal nerve fiber state. All images were acquired with a field of view of $400 \mu \mathrm{m} \times 400 \mu \mathrm{m}$.

\section{QST}

The QST results are summarized in Figure 3 (except PHS and ALL since these were scored dichotomously) and Table 3. Cold detection threshold (CDT) and warm detection threshold (WDT) were significantly reduced values in at least one body location in $76 \%$ and $72 \%$ of patients, with $84 \%$ of patients displaying abnormal thresholds in at least one of these two tests. Two other parameters that were significantly reduced in more than half of the patients were mechanical detection threshold (MDT) in 55\% of patients and vibration detection threshold (VDT) in $71 \%$ of patients. Thus, $71 \%$ of patients had signs of large fiber damage (ie, loss of function of VDT).

\section{Patient phenotypes}

We defined four subgroups of patients, based on the presence or absence of pathological IENFD and/or abnormalities in 

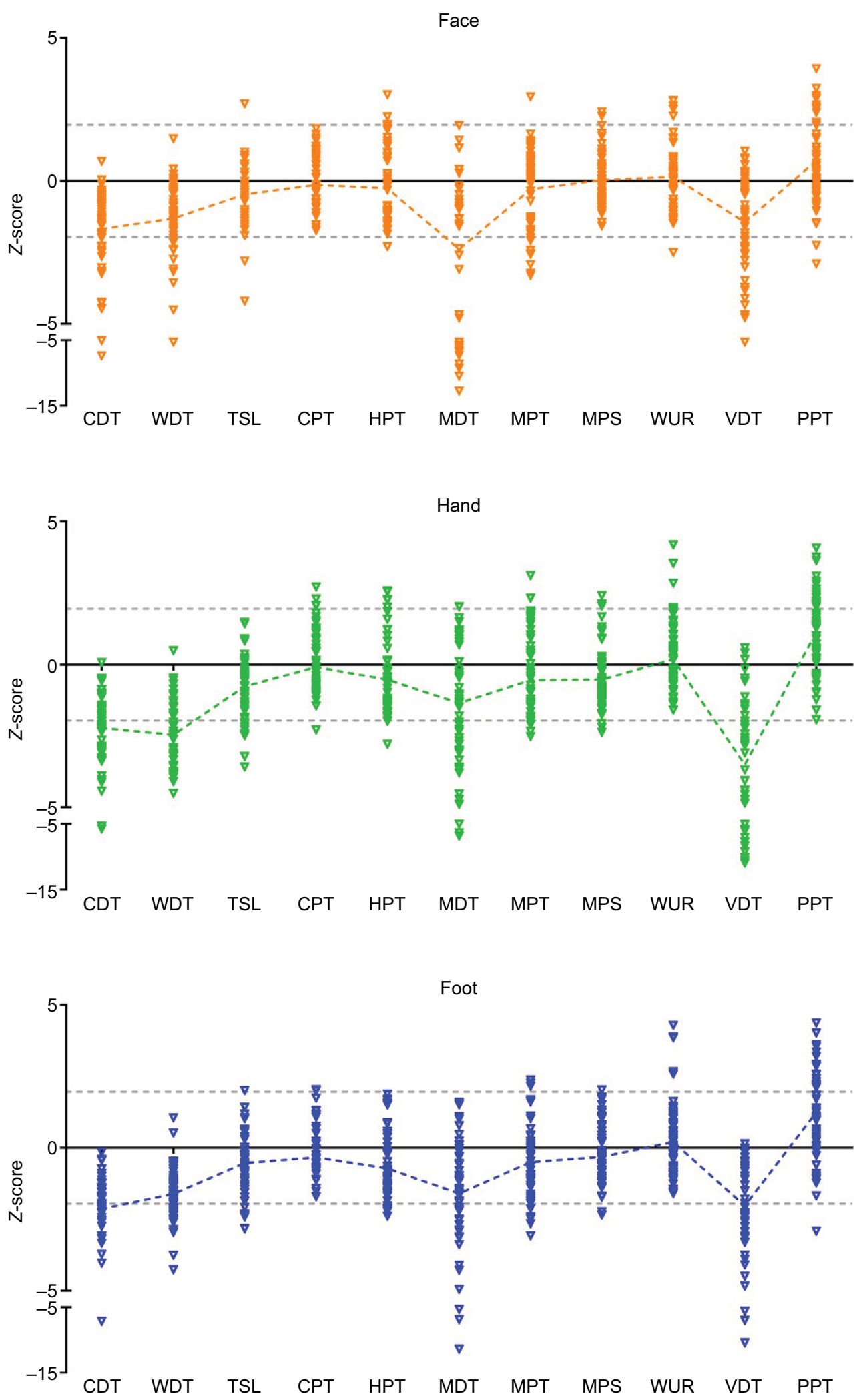

Figure 3 Quantitative sensory test results on three locations of sarcoidosis patients.

Note: Gray dotted lines represent the $95 \%$ confidence interval of the normalized Z-scores.

Abbreviations: CDT, cold detection threshold; CPT, cold pain threshold; HPT, heat pain threshold; MDT, mechanical detection threshold; MPS, mechanical pain sensitivity; MPT, mechanical pain threshold; PPT, pressure pain threshold; VDT, vibration detection threshold; TSL, thermal sensory limen; WDT, warm detection threshold; WUR, wind-up ratio. 
CCM parameters. The four subgroups consisted of a group with decreased IENFD, with and without abnormalities in CCM parameters, and a group with normal IENFD, with and without abnormalities in CCM parameters (Figure 4). The distribution across the phenotypes was as follows: phenotype A, $12 \%(n=7)$ decreased IENFD and abnormalities in CCM parameters; phenotype B, 16\% $(n=9)$ decreased IENFD and normal CCM parameters; phenotype C, 33\% $(n=19)$ normal

Table 3 Average quantitative sensory testing scores and number and percentage of patients with dysesthesias, compared to ageand sex-matched reference values

\begin{tabular}{lll}
\hline Parameter & $\begin{array}{l}\text { Loss of } \\
\text { function, n (\%) }\end{array}$ & $\begin{array}{l}\text { Gain of } \\
\text { function, n (\%) }\end{array}$ \\
\hline Cold detection threshold & $44(76)$ & - \\
Warm detection threshold & $42(72)$ & - \\
Thermal sensory limen & II (19) & $2(3)$ \\
Paradoxical heat sensations & $22(38)$ & - \\
Cold pain threshold & $2(3)$ & $4(7)$ \\
Heat pain threshold & $9(16)$ & $7(12)$ \\
Mechanical detection threshold & $32(55)$ & - \\
Mechanical pain threshold & $15(26)$ & $5(9)$ \\
Mechanical pain sensitivity & $4(7)$ & $3(5)$ \\
Vibration detection threshold & $4 I(71)$ & - \\
Pressure pain threshold & $3(5)$ & $30(52)$ \\
Temporal summation (wind-up) & - & $12(21)$ \\
Dynamic mechanical allodynia & - & $14(24)$ \\
\hline
\end{tabular}

Notes: The prevalence of paradoxical heat sensations and dynamic mechanical allodynia was recorded in a dichotomous fashion (present or not present). Measurements were taken from three locations: on the cheekbone (face) and the dorsal surface of hand and foot. Reference values based on Magerl et al. ${ }^{24}$
IENFD and abnormalities in CCM parameters; phenotype D, $40 \%(n=23)$ normal IENFD and normal CCM parameters (Figure 4).

Subgroup analysis revealed no differences among phenotypes in age $(P=0.90), \mathrm{BMI}(P=0.82)$, sarcoidosis disease duration $(P=0.24)$, average daily pain score $(P=0.14)$, or QST parameter values.

\section{Discussion}

To characterize sarcoidosis patients with neuropathic pain, we performed skin biopsies, CCM, and standardized sensory testing. We defined four distinct phenotypes based on abnormalities in IENFD and CCM with overlap (or agreement) in the presence (phenotype A) or absence (phenotype D) of changes in both CCM and IENFD in 12 and $40 \%$ of patients, respectively. Additionally, the principal sign of small fiber dysfunction, reduced thermal detection thresholds during sensory testing, was present in $84 \%$ of patients. The primary conclusion of our study is that small fiber pathology is present in the majority of our sarcoidosis patients, albeit that different diagnostic tests yield different quantitative results.

\section{Small fibers: CCM versus IENFD}

Absence of agreement between IEFND and CCM indices has been reported before in diabetes patients, ${ }^{25}$ although other studies did find a clear correlation between these two measures in sarcoidosis and diabetes. ${ }^{10,26}$ A regression analysis

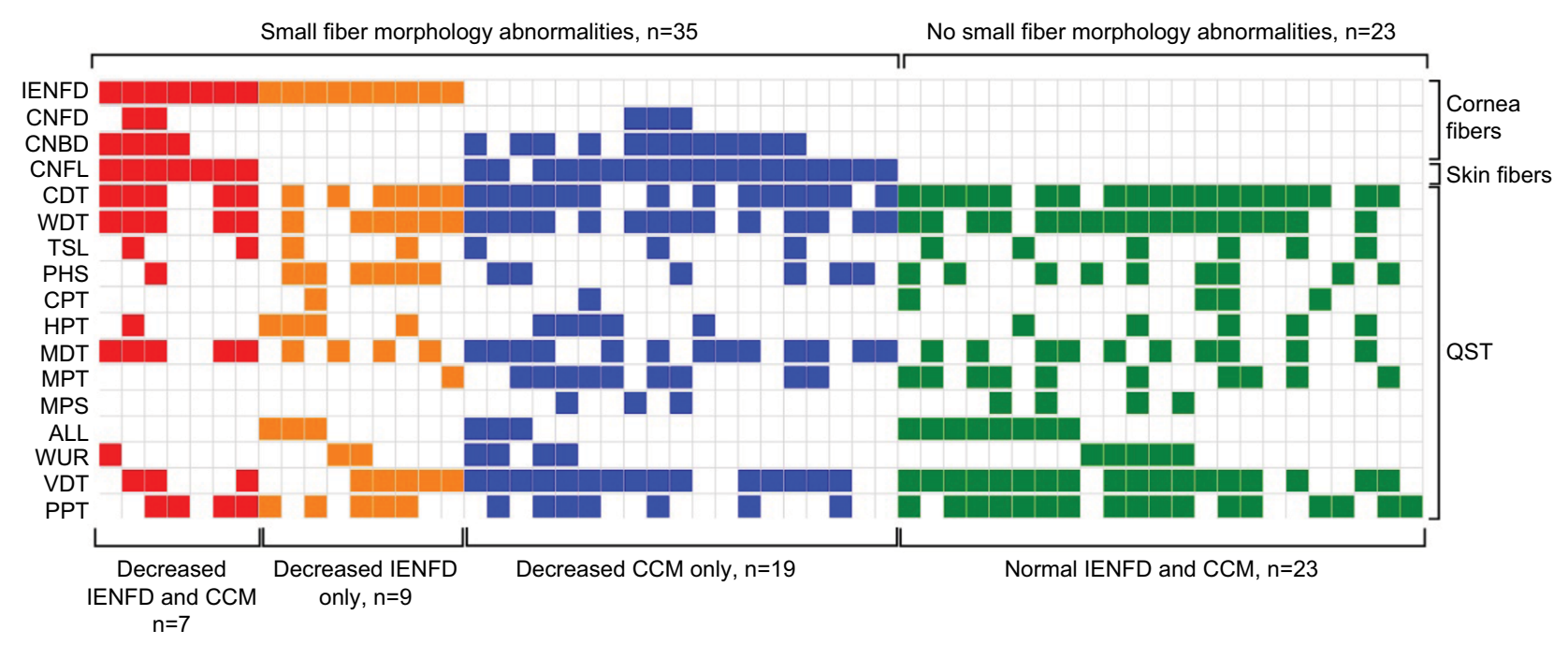

Figure 4 Neuropathic pain phenotypes of sarcoidosis patients.

Notes: Four phenotypes were constructed based on the presence or absence of small fiber neuropathy measured by CCM or skin biopsies. Red: small fiber morphology abnormalities by CCM and skin biopsy, phenotype A. Orange: small fiber morphology abnormalities by skin biopsy only, phenotype B. Blue: small fiber morphology abnormalities by CCM only, phenotype C. Green: no small fiber morphology abnormalities, phenotype D. ALL, dynamic mechanical allodynia.

Abbreviations: CCM, cornea confocal microscopy; CDT, cold detection threshold; CNBD, corneal nerve branching density; CNFD, corneal nerve fiber density; CNFL, corneal nerve fiber length; CPT, cold pain threshold; HPT, heat pain threshold; IENFD, intraepidermal nerve fiber density; MDT, mechanical detection threshold; MPS, mechanical pain sensitivity; MPT, mechanical pain threshold; PHS, paradoxical heat sensations; PPT, pressure pain threshold; QST, quantitative sensory testing; TSL, thermal sensory limen; VDT, vibration detection threshold; WUR, wind-up ratio; WDT, warm detection threshold. 
of CCM versus IENFD data in our population showed a lack of correlation between the two measures (Figure 5). Our results suggest that SFN based on IENFD occurred in just 16 patients $(28 \%)$. However, it may well be that small fiber pathology remained undetected in the skin biopsies obtained from the distal leg. Possibly many of our patients suffer from nonlength-dependent SFN or a mix of lengthand nonlength-dependent distal axonopathies. ${ }^{27}$ For example, patients with abnormalities in CCM but not in skin biopsies or patients without any abnormalities in CCM and normal IENFD may suffer from a nonlength-dependent form of small fiber pathology. Alternatively, it may well be that the abnormalities observed in CCM may precede abnormalities in skin biopsies as has been suggested for diabetes mellitus ${ }^{26}$ or that large fibers are involved at other anatomic locations. To attain detailed phenotypic information of patients, small fibers should be quantified using noninvasive CCM measures as well as skin biopsies.

\section{QST}

We used QST to complement the phenotyping based on skin biopsies and CCM. Where skin biopsies and CCM allow quantification of small nerve fibers, QST provides qualitative assessment of small and large nerve fibers. QST revealed that both small and large fibers were affected in our population (Table 3), indicating that neuropathic pain in sarcoidosis may often be a form of polyneuropathy rather than an exclusive small fiber problem. Large fiber problems may, for example, be the cause of pain in patients with phenotype $\mathrm{D}$. In addition, QST abnormalities were detected in some patients on multiple tested anatomic locations, ie, on the cheekbone (face) and the dorsal surface of hand and/or foot, again indicating a nonlength-dependent, more widespread distribution of sensory disturbances.

\section{Phenotyping: a comparison with the literature}

We previously showed that another chronic pain state with a much more complex and less-well understood pathophysiological mechanism, fibromyalgia, can be described according to the four phenotypes that we present here. ${ }^{1}$ Also, other studies have stratified chronic pain patients according to QST and pain questionnaire outcomes and identified homogeneous patient groups comparable to ours. For example, in a rather heterogeneous population of neuropathic pain patients (central post stroke pain, posttraumatic peripheral pain, painful HIV neuropathy, and painful diabetic peripheral neuropathy), four clusters with distinct pain phenotypes were detected based on QST (cold-evoked pain and touch-evoked pain) and questionnaires (provoked, deep, and pinpoint pain). Despite the different determinants for subgroup identification, the results are in agreement with our findings that homogeneous patterns of symptoms and pathology could be identified irrespective of the underlying disease. ${ }^{1}$ Similarly, in a large survey of 2,100 patients with diabetic painful neuropathy or postherpetic neuralgia, patient-reported outcomes from the PainDETECT questionnaire were used to identify homogeneous subgroups of patients. ${ }^{28}$ Five subgroups were identified with notable differences in quality of pain based on the description of pain (burning and prickling), the occurrence of attacks, pain responses to thermal or pressure stimuli, and the presence of allodynia and numbness. Although the distribution of subgroups differed between postherpetic neuralgia and diabetic polyneuropathy, all subgroups showed relevant numbers of patients. Altogether, these results demonstrate that defining somatosensory phenotypes can identify subgroups of patients with homogeneous signs and symptoms across different disease states. Since the presence of these sensory symptoms is likely related to specific pain-generating
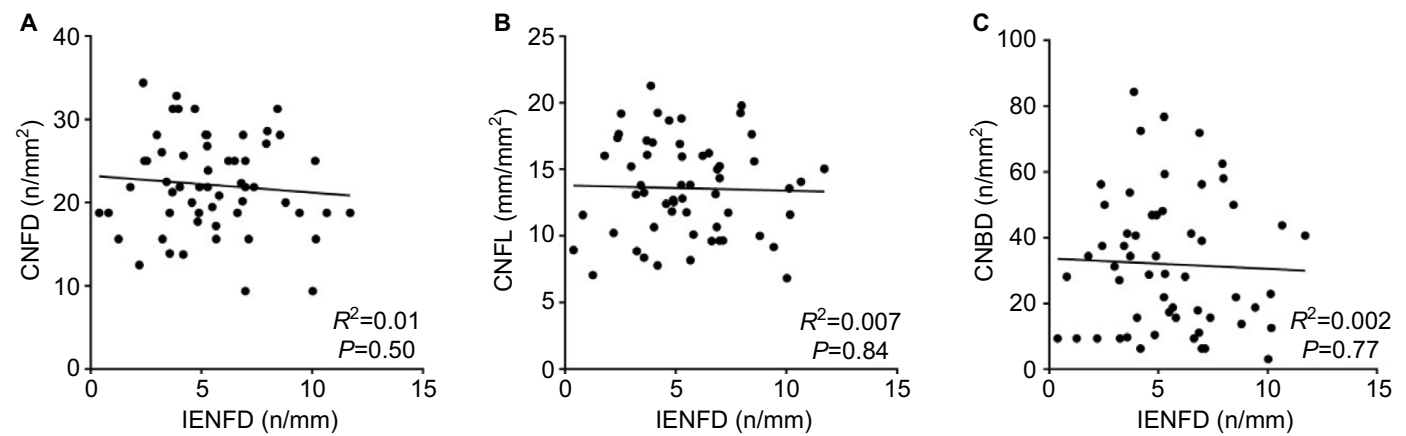

Figure 5 Correlations between CCM parameters CNFD (A), CNFL (B), CNBD (C), and IENFD assessed in skin biopsies from patients with sarcoidosis and neuropathic pain. Note: Linear regression coefficients and associated $P$-values are shown in the bottom of each panel.

Abbreviations: CCM, cornea confocal microscopy; CNBD, cornea nerve branching density; CNFL, cornea nerve fiber length; IENFD, intraepidermal nerve fiber density. 
pathophysiological mechanisms, these subgroups probably will show differential responses to analgesic treatment.

In the current study, we do not report treatment efficacy in the identified patient phenotypes. We may however speculate that patients with evident small nerve fiber pathology would benefit especially from treatment aimed at restoration of small fiber damage and stabilization of the central nervous system inflammatory response to peripheral nerve damage, irrespective of their underlying disease. One such treatment is ARA290, an erythropoietin analog acting at the innate repair receptor, which restores peripheral nerve defects, inhibits spinal cord inflammation, and reduces neuropathic symptoms. Indeed, we showed previously that ARA290 is effective in sarcoidosis and diabetes patients with neuropathic pain. ${ }^{16}$

\section{Study limitations}

There are some limitations to our study. 1) We included patients with a pain score of $>5$ and typical symptoms of neuropathic pain. Consequently, our phenotypes are limited to individuals with these more advanced disease characteristics. Including a more diverse group of patients in terms of disease progression might have offered a better understanding of the development of nerve fiber dysfunction and morphological changes. Nevertheless, we feel that this clinically more severe patient cohort represents an important target of stratification for therapeutic optimization. 2) Since the pain symptoms of our patients were severe, their pain medication was maintained. This might have affected some of the QST results, especially the pain thresholds. 3) Where other studies make a distinction between affected and unaffected areas, we applied all stimuli on predefined locations on the body. Consequently, for some patients, the test location was an affected area, but for others, it was not. 4) QST is a semisubjective test tool. Hence, a lack of correlation between laboratory and clinical findings possible due to the influence of psychological factors, verbal suggestion, or response bias could have occurred. ${ }^{29} 5$ ) Finally, in future research, testing could be extended with microneurography or nerve conduction studies to assess nerve fiber functionality in a more objective manner. Although this will be more time-consuming, it will allow more complete phenotypes and possibly a better correlation between clinical symptoms and laboratory findings. We argue for multimodal assessment of patients, including at least skin biopsies, CCM, and QST measures, to construct detailed somatosensory phenotypes.

\section{Conclusion}

We identified four somatosensory phenotypes in sarcoidosis patients based on IENFD and CCM parameters. The four identified phenotypes suggest that our group of sarcoidosis patients constitute a population with nonlength-dependent or a mixed form of length- and nonlength-dependent SFN. Additionally, we cannot exclude that in some patients without abnormalities in small fibers, large fiber neuropathy played an important mechanistic role in their chronic pain state.

\section{Acknowledgments}

The authors thank the Faculty of Medical and Human Sciences of the University of Manchester, UK, for kindly providing the ACCMetrics software to quantify the confocal images. This study was supported in part by Araim Pharmaceuticals, Inc.

\section{Author contributions}

All authors contributed toward protocol writing, data acquisition, data analysis, drafting, and critically revising the paper, gave final approval of the version to be published, and agree to be accountable for all aspects of the work.

\section{Disclosure}

AD is a chairman of the Leiden University Medical Center's Institutional Review Board. He was not involved in the ethical review of this study. The authors report no other conflicts of interest in this work

\section{References}

1. Oudejans L, He X, Niesters M, Dahan A, Brines M, van Velzen M. Cornea nerve fiber quantification and construction of phenotypes in patients with fibromyalgia. Sci Rep. 2016;6:23573.

2. Dahan A, Olofsen E, Niesters M. Pharmacotherapy for pain: efficacy and safety issues examined by subgroup analyses. Pain. 2015;156(suppl 1): S119-S126.

3. Dworkin RH, O'Connor AB, Audette J, et al. Recommendations for the pharmacological management of neuropathic pain: an overview and literature update. Mayo Clin Proc. 2010;85(3 suppl):S3-S14.

4. Javed S, Alam U, Malik RA. Burning through the pain: treatments for diabetic neuropathy. Diabetes Obes Metab. 2015;17(12):1115-1125.

5. Watson JC, Dyck PJ. Peripheral neuropathy: a practical approach to diagnosis and symptom management. Mayo Clin Proc. 2015;90(7):940-951.

6. Drent M, Strookappe B, Hoitsma E, De Vries J. Consequences of sarcoidosis. Clin Chest Med. 2015;36(4):727-737.

7. Heij L, Niesters M, Swartjes M, et al. Safety and efficacy of ARA 290 in sarcoidosis patients with symptoms of small fiber neuropathy: a randomized, double-blind pilot study. Mol Med. 2012;18: 1430-1436.

8. Hoitsma E, Marziniak M, Faber CG, et al. Small fibre neuropathy in sarcoidosis. Lancet. 2002;359(9323):2085-2086.

9. Tavee J, Culver D. Sarcoidosis and small-fiber neuropathy. Curr Pain Headache Rep. 2011;15(3):201-206. 
10. Brines M, Swartjes M, Tannemaat MR, et al. Corneal nerve quantification predicts the severity of symptoms in sarcoidosis patients with painful neuropathy. Technology. 2013;1(1):1-7.

11. Papanas N, Ziegler D. Corneal confocal microscopy: recent progress in the evaluation of diabetic neuropathy. J Diabetes Investig. 2015;6(4): 381-389.

12. Rolke R, Baron R, Maier C, et al. Quantitative sensory testing in the German Research Network on Neuropathic Pain (DFNS): standardized protocol and reference values. Pain. 2006;123(3):231-243.

13. Chen X, Graham J, Dabbah MA, et al. Small nerve fiber quantification in the diagnosis of diabetic sensorimotor polyneuropathy: comparing corneal confocal microscopy with intraepidermal nerve fiber density. Diabetes Care. 2015;38(6):1138-1144.

14. Hoitsma E, De Vries J, Drent M. The small fiber neuropathy screening list: construction and cross-validation in sarcoidosis. Respir Med. 2011;105(1):95-100.

15. Lauria G, Hsieh ST, Johansson O, et al; European Federation of Neurological Societies; Peripheral Nerve Society. European Federation of Neurological Societies/Peripheral Nerve Society Guideline on the use of skin biopsy in the diagnosis of small fiber neuropathy. Report of a joint task force of the European Federation of Neurological Societies and the Peripheral Nerve Society. Eur J Neurol. 2010;17(7):903-912, e944-e909.

16. Dahan A, Dunne A, Swartjes M, et al. ARA 290 improves symptoms in patients with sarcoidosis-associated small nerve fiber loss and increases corneal nerve fiber density. Mol Med. 2013;19:334-345.

17. Lauria G, Bakkers M, Schmitz C, et al. Intraepidermal nerve fiber density at the distal leg: a worldwide normative reference study.J Peripher Nerv Syst. 2010;15(3):202-207.

18. Chen X, Graham J, Dabbah M, Petropoulos I, Tavakoli M, Malik R. An automatic tool for quantification of nerve fibres in corneal confocal microscopy images. IEEE Trans Biomed Eng. 2016;64(4):786-794.

19. Dabbah MA, Graham J, Petropoulos I, Tavakoli M, Malik RA. Dual-model automatic detection of nerve-fibres in corneal confocal microscopy images. Med Image Comput Comput Assist Interv. 2010;13(pt 1):300-307.
20. Dabbah MA, Graham J, Petropoulos IN, Tavakoli M, Malik RA. Automatic analysis of diabetic peripheral neuropathy using multi-scale quantitative morphology of nerve fibres in corneal confocal microscopy imaging. Med Image Anal. 2011;15(5):738-747.

21. Petropoulos IN, Manzoor T, Morgan P, et al. Repeatability of in vivo corneal confocal microscopy to quantify corneal nerve morphology. Cornea. 2013;32(5):e83-e89.

22. Petropoulos IN, Alam U, Fadavi H, et al. Rapid automated diagnosis of diabetic peripheral neuropathy with in vivo corneal confocal microscopy. Invest Ophthalmol Vis Sci. 2014;55(4):2071-2078.

23. Tavakoli M, Ferdousi M, Petropoulos IN, et al. Normative values for corneal nerve morphology assessed using corneal confocal microscopy: a multinational normative data set. Diabetes Care. 2015;38(5):838-843.

24. Magerl W, Krumova EK, Baron R, Tolle T, Treede RD, Maier C. Reference data for quantitative sensory testing (QST): refined stratification for age and a novel method for statistical comparison of group data. Pain. 2010;151(3):598-605.

25. Ziegler D, Papanas N, Zhivov A, et al; German Diabetes Study (GDS) Group. Early detection of nerve fiber loss by corneal confocal microscopy and skin biopsy in recently diagnosed type 2 diabetes. Diabetes. 2014;63(7):2454-2463.

26. Quattrini C, Tavakoli M, Jeziorska M, et al. Surrogate markers of small fiber damage in human diabetic neuropathy. Diabetes. 2007;56(8):2148-2154.

27. Khoshnoodi MA, Truelove S, Burakgazi A, Hoke A, Mammen AL, Polydefkis M. Longitudinal assessment of small fiber neuropathy: evidence of a non-length-dependent distal axonopathy. JAMA Neurol. 2016;73(6):684-690.

28. Baron R, Tolle TR, Gockel U, Brosz M, Freynhagen R. A cross-sectional cohort survey in 2100 patients with painful diabetic neuropathy and postherpetic neuralgia: differences in demographic data and sensory symptoms. Pain. 2009;146(1-2):34-40.

29. Truini A, Cruccu G. How diagnostic tests help to disentangle the mechanisms underlying neuropathic pain symptoms in painful neuropathies. Pain. 2016;157(suppl 1):S53-S59.

\section{Journal of Pain Research}

\section{Publish your work in this journal}

The Journal of Pain Research is an international, peer reviewed, open access, online journal that welcomes laboratory and clinical findings in the fields of pain research and the prevention and management of pain. Original research, reviews, symposium reports, hypothesis formation and commentaries are all considered for publication

\section{Dovepress}

The manuscript management system is completely online and includes a very quick and fair peer-review system, which is all easy to use. Visit http://www.dovepress.com/testimonials.php to read real quotes from published authors. 